\title{
RSBA-RESOURCE SHARING BEAMFORMING ACCESS FOR 5G-mMTC
}

\author{
Carles Diaz-Vilor, Ana I. Perez-Neira, Senior Member, IEEE, Miguel A. Lagunas, Fellow Member, IEEE
}

\begin{abstract}
Providing connectivity in massive Machine Type Communications (mMTC) poses interesting challenges. In this paper, we propose and study a time diversity beamformer for reception in grant-free access in order to support massive uplink connectivity within the paradigm of the Internet of Things (IoT). The proposed access technique does not require channel estimation or prior network scheduling and provides benefits in terms of Probability of Collision and/or achievable Rate. The combination of our frame structure proposal with spatial signal processing at the receiver side yields a reduction on the probability of collision between devices, which, ultimately, implies that more users can access to the media.
\end{abstract}

Index Terms-mMTC, NOMA, Grant-Free, Repetition division multiple access (RDMA), 5G, Beamforming, OFDMA, DFT-SOFDM

\section{INTRODUCTION}

$\mathbf{M}$ ACHINE-type-communications are considered an important technology in the next generation of wireless networks, and are foreseen to form the backbone of the upcoming automated society. However, actual networks are not prepared to support such traffic, and even less the case of mMTC, which require simultaneous connections of a massive number of low-data-rate devices [1], [2]. One promising approach to deal with mMTC is to use Grant-Free (GF) transmissions [3], [4], [5], where each active device directly transmits its packets to the gNB (next generation node base station) without waiting for any permission, that is, directly from idle state. Therefore, GF may yield to much more simple schemes; thus tailored for IoT transmission, apart from avoiding many of the delays that may occur in Grant-Based (GB) access [6], [7]. This work focuses on GF schemes. In particular, we will assume that the multiple access (MA) is done in an autonomous way, [8], which is the simplest approach. However, this is more challenging for the design of the receiver [9], [10], [11] and [12], because nothing is known a priori in terms of resource scheduling or other important parameters for channel estimation.

The other main focus of this work is the management of massive MA, with the main objective of having a small number of collisions [13]. To increase the number of UE accessing the

This work has received funding from the Spanish Ministry of Science, Innovation and Universities under project TERESA-TEC2017-90093-C3-1-R (AEI/FEDER,UE); and from the Catalan Government (2017 SGR 891 and 2017 SGR 1479)

C. Diaz Vilor and A.I. Perez Neira are with the Dept. of Signal and Communications Theory, of the Universitat Politecnica de Catalunya (UPC) A I. Perez-Neira and Miguel A. Lagunas are with CTTC, Spain. Contact e-mail: aperez@cttc.es. medium, recent work has been done in the so-called Resource Share Multiple Access (RSMA) or Non-Orthogonal Multiple Access (NOMA) techniques [14], [15], [16], which can be divided mainly into two types: Power-Domain NOMA (PNOMA) and Signature- Based NOMA (S-NOMA). In the former users are allocated power considering their channel conditions, while the latter spreads the data stream over the resources in a particular manner trying to overcome the nonorthogonality. This signature thus serves as device identification. These schemes increase spectral efficiency and the number of served devices through the transmission of the nonorthogonal PRACH (Physical Random Access Channel) and PUSCH (Physical Uplink Shared Channel or data transmission) even in the same OFDM symbol of the device. GF S-NOMA allows asynchronous, non-orthogonal contentionbased access that is well suited for sporadic uplink transmissions of small data bursts, common in IoT applications. In order to support a large number of devices, this work proposes to incorporate spatial diversity or beamforming in a blind way, that is, without the need of any additional pilot or training sequence. We call it resource sharing beamforming access or RSBA. This is different from existing works such as that in [17]. We note that New Radio (NR) is the first generation of wireless standard that considers the spatial dimension in a feasible and practical way. This paper stresses this fact and incorporates, with a lean design, spatial diversity multiple access to the RSMA or NOMA schemes that have been proposed in the literature.

Many S-NOMA schemes have been proposed (i.e., to design efficient signatures, different operations, such as linear spreading, multi-dimensional modulation, interleaving, and scrambling can be employed [14]). Among them, in this paper we will assume that users make use of the Repetition Division Multiple Access (RDMA). This scheme employs different simple cyclic-shift repetition patterns at the symbol-level to design device-specific signatures. The cyclic-shift repetition behaves as a randomizer (interleaver), and provides both time and frequency diversity. In fact, in this paper we propose a technique to use the redundancy that RDMA presents in order to create blind beamformers. We note that there are other key factors that need to be managed in upper layers with the aim of having a reliable link. Nevertheless, this paper focuses only on the physical layer design and performance.

The majority of existing wireless communication systems need to estimate the channel (using reference signals) to correctly detect the transmitted symbols [18], [19], [20]. However, with RSMA schemes channel estimation becomes 
more difficult than with orthogonal access schemes due to the higher interference as resources are reused. When the number of devices in the network becomes large, such as in mMTC applications, this task becomes even more difficult due to the increase number of collisions. Therefore, the conventional procedure, whenever spatial combining is implemented, that consists in estimating the channel first and then combine cannot be followed. In fact, the contribution of this paper is a beamforming technique that does not require a previous channel estimation. It harnesses the spatial combining gain as early as possible in order to alleviate collisions and make channel estimation easier with increased Signal to Interference and Noise Ratio (SINR); thus, leading to better demodulation performance.

The remainder of the paper is organized as follows. Section II introduces the signal model at the receiver. In Section III, we present our novel scheme or RSBA. Afterwards, Section IV evaluates it for mMTC scenarios and provides simulations in terms of Probability of Collision between users getting the available resources and achievable Rate. Finally, the last section is devoted for the conclusions of the work.

\section{Signal Model}

Let us consider the uplink physical channel with $N_{u}$ devices or user equipment (UE), each one transmitting the so-called DFT Spread OFDM (DFT-S-OFDM) in NR. Our purpose is to beamform or directionally transmit over mmWave bands to support mMTC. Therefore, the gNB can be equipped with a large number of antennas, denoted by $N_{a}$. Since each UE transmits its information over a wireless multipath channel, we denote the channel taps by $\boldsymbol{h}_{i}=\left[h_{i, 0} h_{i, 1} \ldots h_{i, L_{i}-1}\right] \in$ $\mathbb{C}^{L_{i} \times 1}$ for $i=1,2, \ldots, N_{u}$. In our simulations we have considered the Extended Pedestrian A model that is defined by 3GPP, but this is without loss of generality since our system also works if the UEs present heterogeneous delay profiles or channels. The snapshot at the gNB is given by:

$$
\begin{gathered}
\boldsymbol{y}(n)=\sum_{i=1}^{N_{u}} \boldsymbol{S}_{\boldsymbol{h}_{i}}\left(\boldsymbol{h}_{i}^{*} \circ \boldsymbol{x}_{i}(n)\right)+\boldsymbol{n}= \\
=\sum_{i=1}^{N_{u}}\left[\boldsymbol{s}_{h_{i, 0}} \boldsymbol{s}_{h_{i, 1}} \ldots \boldsymbol{s}_{h_{i, L-1}}\right]\left(\boldsymbol{h}_{i}^{*} \circ \boldsymbol{x}_{i}(n)\right)+\boldsymbol{n}
\end{gathered}
$$

where $\circ$ denotes the Hadamard product and $s_{h_{i, l}} \in \mathbb{C}^{N_{a} \times 1}$ stands for the steering vector of user " $i$ " coming from the angle associated with the respective multipath of the channel. Compacting all the steerings from a device we obtain matrix $\boldsymbol{S}_{\boldsymbol{h}_{i}} \in \mathbb{C}^{N_{a} \times L_{i}}$. In general, a steering vector of a linear uniform array whose direction of arrival (DOA) is given by $\theta$, is formulated as $s(\theta)=$

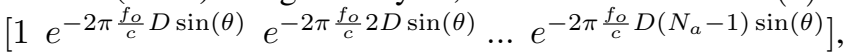
where $D$ represents the distance between antennas, which we design as $\frac{\lambda}{2}, f_{o}$ is the carrier frequency and $c$ is the speed of light. As we are considering the general case of a channel with multipath, the steering vector is the one with

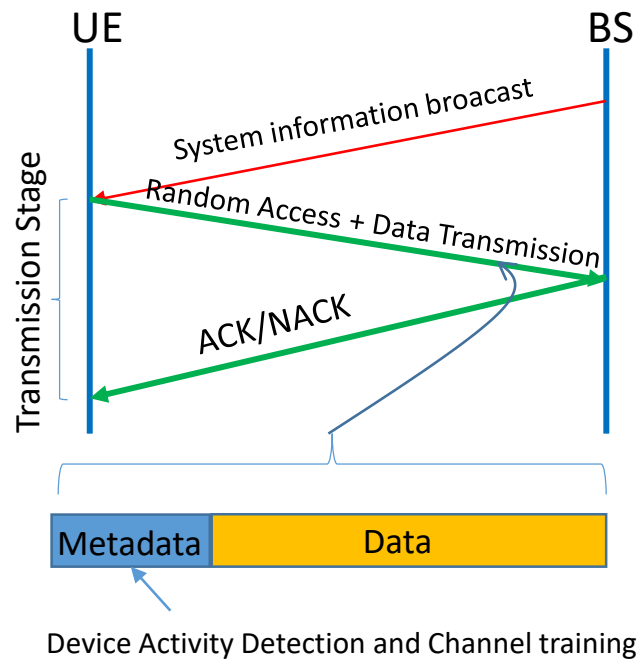

Fig. 1. Grant Free access: Data is directly transmitted together with metadata without waiting for the grant/schedule from BS.

the DOA corresponding to the stronger arriving ray. Vector $\boldsymbol{x}_{i}(n)$ contains the data symbols or the training sequence, as Figure 2 indicates. To simplify notation we assume that users are synchronized, although the beamformer that is presented in the next section can also perform in asynchronous mode. Finally, $\boldsymbol{n} \sim N\left(\mathbf{0}, N_{o} \boldsymbol{I}\right) \in \mathbb{C}^{N_{a} \times 1}$ represents the Additive White Gaussian noise (AWGN).

Although the proposed beamformer does not require any training sequence, we need to embed two kinds of reference signals in the transmitted frames. The first one is the PRACH to transmit a random-access preamble from the UE to indicate to the gNB a random-access attempt and to assist the gNB to adjust the uplink timing of the UE, among other parameters. The second reference signal is used by the gNB to produce channel estimates for demodulation of the associated physical channel (so-called DMRS or demodulation reference signal). In GF access, UE signature for activity detection (PRACH), channel training (DMRS) and information data are sent in the same packet or frame (see Figure 1). Next section explains the rationale behind the proposed beamforming access.

\section{RSBA-RESOURCE SHARING BEAMFORMING}

The proposed RSBA is a GF access and its goal is to reduce the probability of collision in RSMA without increasing signaling overhead or the UE complexity. We note that, for the first time in wireless standards, NR features a highly flexible and unified channel state information framework that is much more advanced and dynamic than in LTE, and which is really prepared to allow different antenna deployments, depending on which part of the spectrum is used for its operation. For instance, for high frequencies (including those in the mmWave range), a large number of antennas can be employed, which increases the capability for beamforming, being the scenario under consideration in this paper. Beamforming improves the overall radio environment of a cell by limiting interference to 


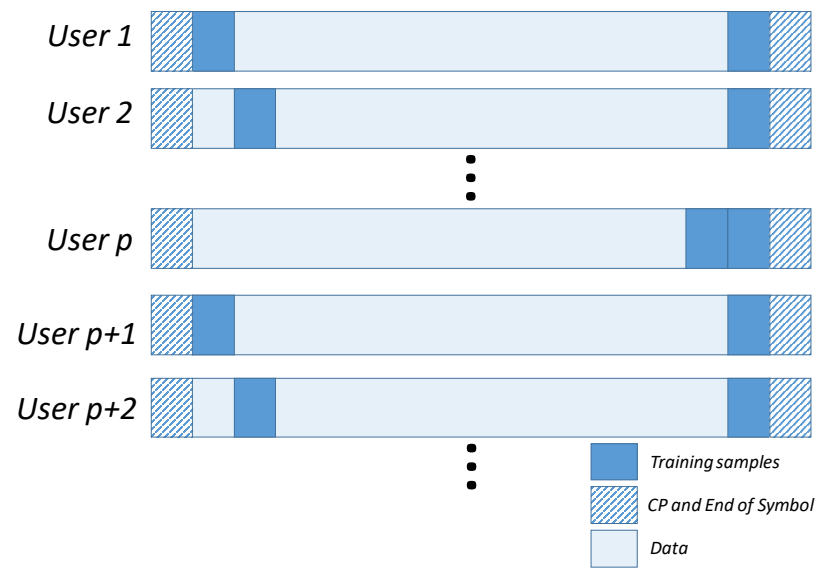

Fig. 2. Frame structure for the RSBA scheme, where each user repeats in a particular time slot its training sequence. Also, to preserve the circularity of the channel, we make use of the CP.

small fractions of the entire space around a transmitter and likewise limiting the impact of interference on a receiver. It can be conceived as a key tool to allow zero broadcast in NR; thus, saving energy. In this respect, to improve energy and overhead efficiency we further develop the $5 \mathrm{G}$ concept of usercentric beamforming, that relieves the UE from monitoring and processing reference signals from all nearby gNBs. This contrasts with LTE.

The user-centric strategy fits very well within the grant-free random access, where each active UE directly transmits to the gNB without waiting for any permission. Even though S-NOMA is a new promising approach to deal with high density scenarios, such architectures do not contemplate using the signature to separate users in space, which is the purpose of RSBA. In order to implement it, we take advantage of the RDMA scheme, which assigns distinct cyclic shift repetition patterns in the time or frequency domain to the users.

As PRACH we propose RDMA. As the information data or PUSCH is a DFT-S-OFDM, for the DMRS we propose to use Zadoff-Chu sequences. Like in LTE, Zadoff-Chu sequences are used, due to their good properties, including constant amplitude before and after DFT, zero cyclic auto-correlation and low cross-correlation. This training signal can be inserted in the repetition slots, as Figure 2 illustrates. For the beamformer design that we are going to propose, any other reference signal with the mentioned properties can be used, as long as it meets that its correlation with the data is zero, which in general is true. We note that the repetition pattern is in time. If we consider an $N$-point DFT-S-OFDM signal, we add the reference sequence (of length $T$ samples) into two different parts. All users can have the same reference signal, as commented before. The reason for adding the redundancy in time, instead of reserving some frequency sub-carriers to send pilot signals, is because whenever the redundancy occupies $T$ frequencies, the associated rate $\frac{N-T}{N}$ is lower than if we add the redundancy in time, which is $\frac{N}{N+T}$.

With the proposed repetition pattern of Figure 2, a beam- former $\boldsymbol{b}_{i}$ can be designed for each UEi, such that the following quadratic cost function is minimized:

$$
\begin{array}{ll} 
& \min _{\boldsymbol{b}_{i}} \mathbb{E}\left\{\left|\boldsymbol{b}_{i}^{H} \boldsymbol{x}_{1}-\boldsymbol{b}_{i}^{H} \boldsymbol{x}_{2}\right|^{2}\right\} \\
\text { s.t. } & 2 \Re\left\{\mathbb{E}\left\{\boldsymbol{b}_{i}^{H} \boldsymbol{x}_{1} \boldsymbol{x}_{2}^{H} \boldsymbol{b}_{i}\right\}\right\}=\gamma
\end{array}
$$

where $\boldsymbol{x}_{1}$ and $\boldsymbol{x}_{2}$ refer to the first and second block of the repeated symbols, respectively. This time diversity beamformer was designed for frequency diversity purposes in [21]. We stress that these symbols are from a reference signal just to allow the timing synchronization and the posterior channel estimation, but if the only purpose were the design of the beamformer then any data symbol would be valid. The only requirement is that they repeat following the pattern. The constraint avoids the undesired solution, being the null vector, where $\gamma$ is some constant different from zero. Defining $\boldsymbol{R}_{k l}=\mathbb{E}\left\{\boldsymbol{x}_{k} \boldsymbol{x}_{l}^{H}\right\} \forall k, l=1,2$, the solution to such problem is:

$$
\left(\boldsymbol{R}_{11}+\boldsymbol{R}_{22}\right) \boldsymbol{b}_{i}=(1+\lambda)\left(\boldsymbol{R}_{12}+\boldsymbol{R}_{12}^{H}\right) \boldsymbol{b}_{i}
$$

$i=1,2, \ldots, N_{u}$. However, for the case where the set of samples $\boldsymbol{x}_{1}$ contains pilot sequences from more than one user, the combiner will point to more than one device. In these cases, due to the large number of antennas that the gNB can have, the sources will be spatially identified with high resolution; thus, allowing the gNB to estimate their corresponding direction of arrival or DOA. After this DOA estimation stage, and considering a properly calibrated array, the steering vector of each UEi is known, $s_{i}$, and then, different spatial reference beamformers can be applied. For instance in this work we apply the phased array beamformer, that is $\boldsymbol{b}_{s, i}=s_{i}$, where subindex $s$ refers to separate. Note that DOA estimation with phased array is precisely what NR proposes under the beam sweeping mechanism in order to support beamforming for initial access [22]. Due to the large number of antennas, such beam would be narrow enough to consider the channel as only presenting one tap, the one with less attenuation. Therefore, the equivalent channel will be just a complex number: $\boldsymbol{h}_{e q, i}=\boldsymbol{b}_{s, i}^{H} \boldsymbol{s}_{i}$. We note that the beamformer distinguishes the users by their different repetition patterns; therefore, they can employ the same DRMS sequence. Also, if this sequence presents zero autocorrelation, as it is the case in point, then multipath is also diminished by the beamformer. To sum up: the proposed RSBA consists in three steps: 1.Blind beamforming (Eq. (3)); 2.- UEs DOA estimation by identifying the maxima of the blind beamformer; 3.- Form a dedicated beam towards each identified DOA; thus, spatially separating users and avoiding collision even if they present the same repetition pattern; 4.- For each user the channel can be subsequently estimated and the information demodulated.

\section{EVALUATION}

This section discusses the gain that the proposed RSBA provides in terms of probability of collision and achievable rate 


\section{A. Probability of Collision}

First, let us imagine the case where the aforementioned RSBA technique is not used. As we are assuming a GF-MA scheme, a collision will occur if any of the users tries to get the same frequency resources at the same time and with the same repetition pattern. Therefore, we can measure the Probability of Collision in this case, which will be denoted by $P_{C, R D}$.

This probability depends on many parameters, such as the number of devices, $N_{u}$, the bandwidth associated to each user or the total number of subcarriers, and the type of NOMA detector (i.e., single user detector - SUD or, sequential interference canceller -SIC). In case the gNB implements SUD for each user, then $P_{C, R D}$ coincides with the probability that users collide in time and frequency. As discussed earlier, if we can spatially separate users, $P_{C, R D}$ is not such a problem, as the beamformer would separate the colliding signals. Thus, decreasing the probability of collision. If we denote by $P_{C, S}$ the probability of collision in the spatial domain, being a measure of the number of wrong detected sources by the RSBA, the total probability of collision, combining frequency and space, is given by:

$$
P_{C, R S}=P_{C, R D} \cdot P_{C, S}
$$

This probability also depends on the quality of the correlation matrix estimates of (3), which depends on $T$ (i.e., the larger $T$ the better the estimate). Thus, trading-off reliability and rate because this latter is given by $R=\frac{N}{N+T+C_{p}}$, where $C_{p}$ is the lenght of the cyclic prefix.

\section{B. Rate Analysis}

We now formulate the rates that we evaluate in the uplink of $5 \mathrm{G}$ scenarios whose multiple access is based on nonorthogonal procedures. In particular, we will assume the worst case, in which the bandwidth and central frequency associated to each device is the same, UEs simultaneously transmit, and when the gNB implements a SUD for each UE. Hence, there is full frequency domain collision among all of the UEs.

We now write the achievable rate expressions that we are going to evaluate. First, we have denoted by $R_{i, N, B}$ the uplink rate of the $i$-th device using NOMA (subindex $N$ ) with the steering beamformer (subindex $B$ ) pointing that particular UE. Second, to show the gain a spatial combiner provides we also include $R_{i, N}$ which is the rate of a NOMA access such as RDMA without any beamformer. In that case, as we are not multiplexing users in space, the receiver will work with a lower SINR value. Finally, to see which is the gain between NOMA and orthogonal multiple access in frequency (OMA) (subindex $O$ ), both using a spatial combiner, we include $R_{i, O, B}$. Let us define:

$$
\begin{gathered}
S_{i}=\left|\boldsymbol{b}_{s, i}^{H} \boldsymbol{s}_{h_{i, 0}} h_{i, 0}\right|^{2} \\
N_{B}=\frac{P_{n}}{P} \boldsymbol{b}_{s, i}^{H} \boldsymbol{b}_{s, i} \\
M_{i}=\sum_{l=1}^{L_{i}-1}\left|\boldsymbol{b}_{s, i}^{H} \boldsymbol{s}_{h_{i, l}} h_{i, l}\right|^{2}
\end{gathered}
$$

$$
I=\sum_{\forall j \neq i}^{N_{u}} \sum_{l=0}^{L_{j}-1}\left|\boldsymbol{b}_{s, i}^{H} \boldsymbol{s}_{h_{j, l}} h_{j, l}\right|^{2}
$$

where $P$ stands for the transmission power that is used by each UE for the data. From (5) to (8), S, $P_{n}, \mathrm{M}$ and I represent Signal, Noise after beamformer, Multipath interference and Inter-user interference, respectively (all terms normalized by $P)$. We can write the rate expressions as a function of (5), (6), (7) and (8) as follows:

$$
\begin{gathered}
R_{i, N, B}=\left(1-P_{C, R S}\right) \log _{2}\left(1+\frac{S_{i}}{N_{B}+M_{i}+I}\right) \\
R_{i, N}=\left(1-P_{C, R D}\right) \log _{2}\left(1+\frac{\left|h_{i, 0}\right|^{2}}{\frac{P_{n}}{P}+\sum_{l=1}^{L_{i}-1}\left|h_{i, l}\right|^{2}+\sum_{\forall j \neq i}^{N_{u}} \sum_{l=0}^{L_{j}-1}\left|h_{j, l}\right|^{2}}\right) \\
R_{i, O, B}=\frac{1}{N_{u}} \log _{2}\left(1+\frac{S_{i}}{\frac{N_{B}}{N_{u}}+M_{i}}\right)
\end{gathered}
$$

The previous expressions show that the only term not being considered as interference is the ray arriving directly from the LoS path. Power arriving from other directions (including other users in case of a NOMA) is considered as interference.

\section{Numerical Results}

We now compare our proposed scheme with other state-ofthe-art strategies. We provide two sets of results. The first one is related to the Probability of Collision, where we include simulation results showing a reduction on that probability if our scheme is used. Second we evaluate the rates that are shown in IV-B.

Before that, we would like to remark the difficulties of such analysis, as very little work has been done assuming a large number of devices due to the high computational cost of running massive scenarios. Therefore, we think this paper could provide a very first approach and show more qualitatively rather than quantitatively the tendencies in the variables we have measured.

Regarding the study on the Probabilities of Collision, we generated a scenario in which at most there were up to $N_{u}=120$ devices located at different positions, randomly requesting 36 or 48 subcarriers. We averaged 500 independent and identical random realizations for the cases where the DFTS-OFDM symbols are of length $N=1024$ (with a total number of available subcarriers of 601), a value of $T=300$ referred to the training length and different antenna sizes: $N_{a}=\left[\begin{array}{llll}16 & 32 & 64 & 128\end{array}\right]$ with a distance between antenna elements of $\frac{\lambda}{2}$ transmitting over the aforementioned EPA channel. In addition, as we are assuming LoS or outdoors environments, we can ensure that only the ray arriving from the first tap will preserve the direction of the source. The rest of them will have different DOAs and have been parametrized as in WINNER [23]. The gNB applies a SUD for each user, therefore, users with different repetition pattern collide if they share the same 


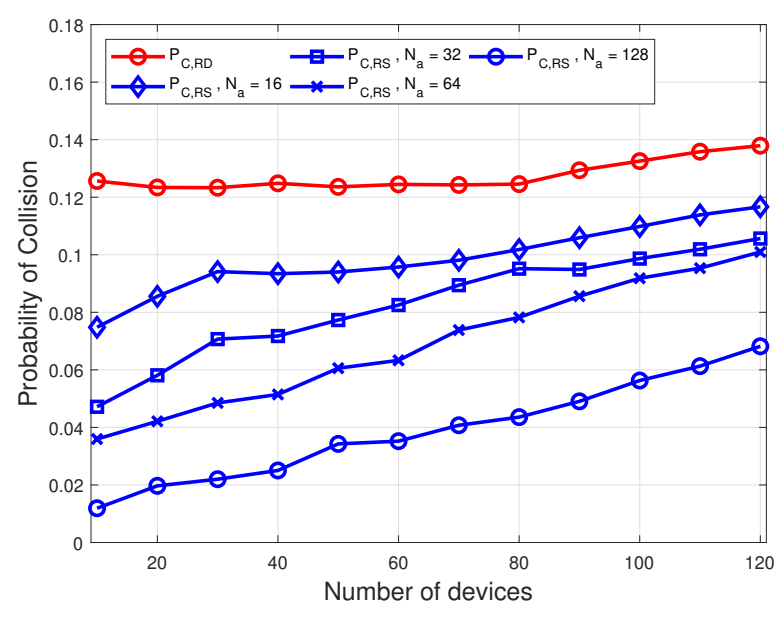

Fig. 3. Probability of collision without (red) and with (blue) spatial diversity, for $N_{a}=\left[\begin{array}{llll}16 & 32 & 64 & 128\end{array}\right], N=1024$ and $T=300$.

time slot and frequency band, unless the spatial dimension is exploited with RSBA. As presented in Figure 3, the expected out-performance of our proposal is accomplished. Note that we compare our RSBA scheme with RDMA (i.e., without beamforming). All curves show an increasing tendency when the number of sources increases. There are two main reasons for that behavior. The first is related to the degrees of freedom, mainly determined by $N_{a}$. The more sources we have, the less degrees of freedom the system has, hence the RSBA beamformer is not able to find all transmitting sources. The second is related to the spatial position of the UEs. If lots of them are near the endfire, due to combiners' nature, they are more difficult to distinguish, yielding to a miss-detection of sources at high elevation angles. Note that this feature is present in all uniform linear arrays. In addition, it is worthy to mention the considerable gain we achieve when increasing the number of antennas at the receiver side. The more antennas we have, the narrower the beams will be and, consequently, if two sources are very close we could distinguish them.

The second set of results we provide is related to the rate a device can achieve. For these experiments, let us assume that such device is always well-detected by the receiver, positioned at 19 of elevation, meaning that $P_{C, R S}=0$, as it would be even harder to simulate all the use cases including the computation of that probability. It must be mentioned that the previous assumption would no longer be valid in case we are measuring the rate of a source located near the endfire, for the reasons we have exposed previously. Again, with a separation between elements of $\frac{\lambda}{2}$ with the same antenna array sizes, 36 subcarriers per device and an SNR value of $15 \mathrm{~dB}$ over an EPA channel, the variation of the rate depending on the number of UEs in the scenario, $N_{u}$, is presented in Figure 4. We can appreciate that all curves have the same tendency, a decrease in the rate when the number of users increases. For the case of NOMA, such decrease is related to interferences, while for OMA, the factor $\frac{1}{N_{u}}$ is the one

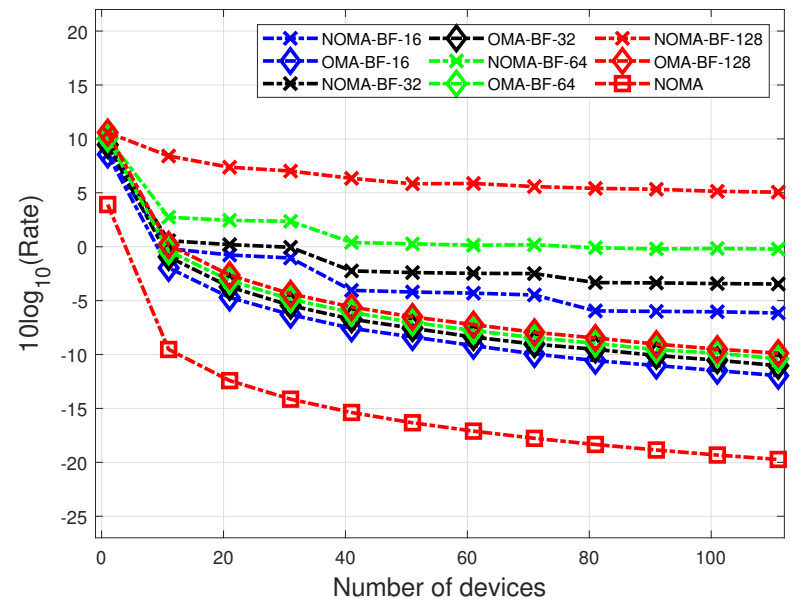

Fig. 4. Variation of the Rate for a user located at 19 of elevation, for different access technologies when the number of devices transmitting increases with an $S N R=15 d B$.

that penalizes more. Therefore, in these cases, it is preferable to deal with interferences enjoying greater bandwidths, than having orthogonality in the frequency domain. Another key point is that small antenna-array sizes are preferred with NOMA than great antenna-array groups with OMA, for the same reason, being the trade-off between bandwidth and $\frac{1}{N_{u}}$. In the plot we denote by "-BF" whenever we incorporate beamforming. For instance, "NOMA-BF" means the RSBA technique that we propose.

Regarding the variation on the rate as a function of the SNR, we also include Figure 5, which, under the same simulation parameters and fixing the number of devices to $N_{u}=111$, we observe two differentiated areas in the NOMA curves. We can appreciate that for low SNR values the slope of the curves is higher than for high SNR values. This means that, in case the system is working in the low SNR regime, what limits the rate is noise, whereas in the high SNR regime there is little increase, as the limit is established by interferences. In addition, the reader can note the gap between NOMA and OMA, which follows the same reasoning as for Figure 4. That is why we also include a theoretical study on the conditions that $R_{i, N, B}$ should fulfill to be greater than $R_{i, O, B}$ when $N_{u} \rightarrow \infty$, being the case of mMTC scenarios. Forcing the RSBA rate to be higher than OMA's, we obtain (12).

$$
\begin{gathered}
\left(1-P_{C, R S}\right) \log _{2}\left(1+\frac{S_{i}}{N_{B}+M_{i}+I}\right) \geq \\
\frac{1}{N_{u}} \log _{2}\left(1+\frac{S_{i}}{\frac{N_{B}}{N_{u}}+M_{i}}\right)
\end{gathered}
$$

After manipulating the previous inequality and taking the $\lim _{N_{u} \rightarrow \infty}$, we obtain that in order to have a higher rate using RSBA the following must be met:

$$
e^{\frac{1}{\left(1-P_{C}, R S\right)}}-1 \geq \frac{S_{i}}{N_{B}+M_{i}+I}
$$




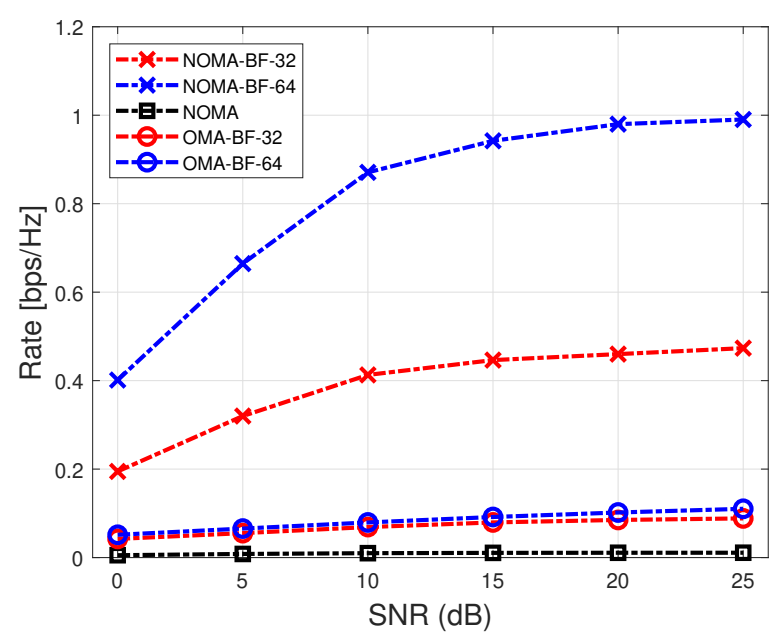

Fig. 5. Variation of the Rate a unique user at 19 of elevation can achieve, with different access technologies and different antenna-array sizes, as a function of the SNR with $N_{u}=111$.

\section{CONCLUSIONS AND FUTURE WORK}

This paper addresses one of the hot topics related to $5 \mathrm{G}$, which is how to deal with mMTC. It is well known that these type of communications will play an important roll in the next generation of wireless communications, being the base of IoT applications and smart-cities/houses among others. During this work we have seen basic aspects of NOMA procedures, which are said to be the ones implemented at the physical layer of the aforementioned applications. In particular, we have proposed a way to take benefit from S-NOMA, and, more precisely RDMA, yielding to what we call: RSBA. If we have a large multi-antenna system, which by design is true for $5 \mathrm{G}$ in mmWave, RSBA allows creating spatial combiners whose ultimate goal is to spatially separate users without requiring the transmission of a training sequence. First, we have shown the superior performance in front of RDMA in terms of probability of collision. This is achieved without requiring additional training data. Second, we have shown the improvements in rate when compared to both, orthogonal access and NOMA without beamforming.

\section{REFERENCES}

[1] C. Bockelmann et. al., "Towards Massive Connectivity Support for Scalable mMTC Communications in 5G networks", IEEE Access, April 2018

[2] C. Bockelmann et. al., "Massive Machine-type Communications in 5G: Physical and MAC-layer solutions", IEEE Communications Magazine, Vol. 54, June 2016

[3] A. Azari, P. Popovski, G. Miao and . Stefanovi, "Grant-Free Radio Access for Short-Packet Communications over 5G Networks", GLOBECOM 2017 - 2017 IEEE Global Communications Conference

[4] M. Masoudi, A. Azari, E. Altug Yavuz and C. Cavdar, "Grant-free Radio Access IoT Networks: Scalability Analysis in Coexistence Scenarios", 2018 IEEE International Conference on Communications (ICC)

[5] C. Wang and Y. Chen and Y. Wu and L. Zhang, "Performance Evaluation of Grant-Free Transmission for Uplink URLLC Services", 2017 IEEE 85th Vehicular Technology Conference (VTC Spring)
[6] S. Sesia, I. Toufik and M. Baker, "LTE The UMTS Long Term Evolution", Wiley, 2011. ISBN: 9780470660256

[7] G. Berardinelli, N. Huda Mahmood, R. Abreu, T. Jacobsen, K. Pedersen, I. Z. Kovcs and P. Mogensen, "Reliability Analysis of Uplink Grant-Free Transmission Over Shared Resources", IEEE Access, Vol. 6, pp 2360223611, 2018

[8] Z. Yuan, Y. Hu, W. Li and J. Dai, "Blind Multi-user Detection for Autonomous Grant-free High-Overloading MA without Reference Signal",

[9] Z. Yuan, C. Yan, Y. Yuan, and W. Li, "Blind Multiple User Detection for Grant-Free MUSA without Reference Signal", 2017 IEEE 86th Vehicular Technology Conference

[10] Y. Du, B. Dong, Z. Chen, X. Wang, Z. Liu, P. Gao and S. Li , "Efficient Multi-User Detection for Uplink Grant-Free NOMA: Prior-Information Aided Adaptive Compressive Sensing Perspective", IEEE Journal on Selected Areas in Communications, July 2017

[11] M. Alam, and Q. Zhang, "A Survey: Non-Orthogonal Multiple Access with Compressed Sensing Multiuser Detection for mMTC', IEEE Journal on Selected Areas in Communications, July 2017

[12] B. Wang, L. Dai, Y. Zhang, T. Mir and J. Li, "Dynamic Compressive Sensing Based Multi-User Detection for Uplink Grant-Free NOMA", IEEE Communications Letters, Vol. 20, November 2016

[13] A. Osseiran, J. F. Monserrat and P. Marsch, "5G Mobile and Wireless Communications Technology", Cambridge University Press, 2016

[14] M. Mohammadkarimi, M. Ahmad Raza and O. Dobre, "Signature-based Non-orthogonal Multiple Access (S-NOMA) for Massive Machine-Type Communications in 5G",

[15] L. Dai and B. Wang and Y. Yuan and S. Han and C. I and Z. Wang, "Nonorthogonal multiple access for 5G: solutions, challenges, opportunities, and future research trends", IEEE Communications Magazine, Vol. 53, no. 9, pp. 74-81, Sept. 2015

[16] Y. Polyanskiy, "A perspective on massive random-access", 2017 IEEE International Symposium on Information Theory (ISIT)

[17] Y. Chi and L. Liu and G. Song and C. Yuen and Y. L. Guan and Y. Li, "Practical MIMO-NOMA: Low Complexity and Capacity-Approaching Solution”, IEEE TWC, Vol. 37, Sept 2018, pp. 6251-6264.”

[18] Y. Liu and Z. Tan and H. Hu and L. J. Cimini and G. Y. Li, "Channel Estimation for OFDM", IEEE Communications Surveys Tutorials, Vol 16, no. 4, pp. 1891-1908, 2014

[19] M. Biguesh and A. B. Gershman, "Training-based MIMO channel estimation: a study of estimator tradeoffs and optimal training signals", IEEE Transactions on Signal Processing, Vol. 54, no. 3, pp. 884-893, 2006

[20] B. Karakaya and H. Arslan and H. A. Cirpan, "Channel Estimation for LTE Uplink in High Doppler Spread”, 2008 IEEE Wireless Communications and Networking Conference

[21] M.A. Lagunas, A.I. Perez Neira, M.G. Amin, and J. Vidal, "Spatial Processing for Frequency Diversity Schemes", IEEE Transactions on Signal Processing, Vol. 48, no. 2, 2000

[22] X. Lin, J. Li, R. Baldemair, T. Cheng, S. Parkvall, D. Larsson, H. Koorapaty, M. Frenne, S. Falahati, A. Grvlen and K. Werner, "5G New Radio: Unveiling the Essentials of the Next Generation Wireless Access Technology", https://arxiv.org/abs/1806.06898

[23] P. Kysti, "WINNER II Channel Models, IST-WINNER Deliverable D1.1.2", European Commission, 2007 